\title{
ON GREEN'S AND NEUMANN'S FUNCTIONS IN THE THEORY OF PARTIAL DIFFERENTIAL EQUATIONS
}

\author{
S. BERGMAN AND M. SCHIFFER ${ }^{1}$
}

Introduction. The purpose of this paper is to describe an effective construction of Green's and Neumann's functions for a general class of linear, second-order partial differential equations of elliptic type in terms of a set of continuously differentiable functions, complete and orthonormal with respect to the domain considered. This result completes a previous paper ${ }^{2}$ by us in which an analogous construction was given for the difference between the Green and Neumann functions. In order to obtain representations of the Green and Neumann functions separately, one had to assume in the former paper the knowledge of a fundamental solution of the differential equation considered. Since it is by no means an easy task to construct for a given linear partial differential equation of second order a fundamental solution, and since the method of this paper permits a direct construction of the Green and Neumann functions without knowledge of such a fundamental solution, our result seems to constitute a certain advance in the application of orthonormal sets to the theory of linear partial differential equations.

1. Generalities and notations. We choose a finite domain $B$ in the plane $(x, y)$ which is bounded by a set of $M$ closed smooth curves $C_{\nu}(\nu=1,2, \cdots, M)$. We shall denote the boundary of $B$ by $C=\sum_{\nu=1}^{M} C_{\nu}$. Let $P(x, y)$ be a continuous positive function in the closed region $B+C$. Consider the linear partial differential equation of elliptic type

$$
\frac{\partial^{2} \phi}{\partial x^{2}}+\frac{\partial^{2} \phi}{\partial y^{2}}=P(x, y) \phi, \quad P(x, y)>0 .
$$

We associate with this equation the following Dirichlet integral

$$
D\{\phi, \psi\}=\iint_{B}\left(\frac{\partial \phi}{\partial x} \frac{\partial \psi}{\partial x}+\frac{\partial \phi}{\partial y} \frac{\partial \psi}{\partial y}+P \phi \psi\right) d x d y
$$

Presented to the Society, April 26, 1947; received by the editors March 17, 1947

${ }^{1}$ Research paper done under Navy Contract NOrd 8555-Task F at Harvard University. The ideas expressed in this paper represent the personal views of the authors and are not necessarily those of the Bureau of Ordnance.

${ }^{2}$ S. Bergman and M. Schiffer, A representation of Green's and Neumann's functions ..., Duke Math. J. vol. 14 (1947). 
which defines a scalar product for each pair of functions $\phi$ and $\psi$ which are continuously differentiable in $B$ and for which the integrals $D\{\phi, \phi\}$ and $D\{\psi, \psi\}$ taken over $B$ in the Lebesgue sense converge. All functions of this type form a linear space $\Omega$ and the scalar product (2) defines a metric in this space.

For the sake of a concise notation, we shall throughout this paper denote a point $(x, y)$ of the domain $B$ by $Z$ and the corresponding complex number $x+i y$ by $z$. Another point $(u, v)$ of $B$ will be denoted by $W$ and analogously $u+i v=w$; in the same way, $(r, s)=T, r+i s=t$.

A function $S(Z, W)$ is called a fundamental solution of (1) in $B$ if it is a solution of (1) as a function of $Z \in B$ for fixed $W \in B$, which is finite everywhere in $B$ with the exception of the point $W$, where it has a logarithmic singularity. It may be written in the form

$$
S(Z, W)=A(Z, W) \log \frac{1}{|z-w|}+\alpha(Z, W)
$$

where $A(Z, W)$ is a solution of (1) of class $\Omega$ which is symmetric in $Z$ and $W$ throughout the whole domain $B$. We may even require additionally

$$
A(W, W)=1 .
$$

$\alpha(Z, W)$ is finite and continuously differentiable in $Z$ at every point of $B$, but it is in general not a solution of (1). There exists an infinity of fundamental solutions of (1). We distinguish two particular fundamental solutions which are of special interest for our theory:

Green's function $G(Z, W)$ of the domain $B$ with respect to the differential equation (1) is that fundamental solution which vanishes, if, for $W \in B$ fixed, $Z$ converges to the boundary $C$ of $B$.

Neumann's function $N(Z, W)$ of the domain $B$ with respect to the differential equation (1) is that fundamental solution whose normal derivative vanishes on the boundary $C$ of $B$ for fixed $W \in B$, that is,

$$
\frac{\partial N(Z, W)}{\partial n_{z}}=0, \quad Z \in C,
$$

where $n_{z}$ denotes the interior normal to $C$ at the point $Z \in C$.

2. Orthonormal sets of solutions of (1). Let $\left\{\phi_{\nu}(Z)\right\}$ be a set of solutions of (1) each of which belongs to the class $\Omega$. We call this set orthonormalized if it satisfies the conditions

$$
D\left\{\phi_{\nu}, \phi_{\mu}\right\}=\delta_{\nu \mu}, \quad \delta_{\nu \mu}= \begin{cases}1 & \text { if } \nu=\mu, \\ 0 & \text { if } \nu \neq \mu .\end{cases}
$$


Let $\Gamma$ be the subclass of $\Omega$ consisting of all solutions $\phi(Z)$ of (1). We call the system $\left\{\phi_{\nu}(Z)\right\}$ complete with respect to the class $\Gamma$ if every element of $\Gamma$ may be approximated arbitrarily by a linear aggregate of functions $\phi_{\nu}(Z)$ uniformly in every closed subdomain of $B$. If this set is complete and orthonormalized, every solution $\phi(Z)$ of class $\Gamma$ may be developed into a series ${ }^{3}$

$$
\phi(Z)=\sum_{\nu=1}^{\infty} a_{\nu} \phi_{\nu}(Z), \quad a_{\nu}=D\left\{\phi_{\nu}, \phi\right\},
$$

which converges uniformly in every closed subset of $B$.

The series

$$
K(Z, W)=\sum_{\nu=1}^{\infty} \phi_{\nu}(Z) \phi_{\nu}(W)
$$

extended over a complete orthonormalized system $\left\{\phi_{\nu}(Z)\right\}$ converges uniformly in every closed subdomain of $B$ and represents there a symmetric function of $Z$ and $W$ which is in each variable a solution of (1). $K$ is called the kernel function of the orthonormal set $\left\{\phi_{\nu}(Z)\right\}$.

By means of (5), (6) and (7), one proves easily that for every element $\phi(Z)$ of class $\Gamma$

$$
\phi(Z)=D_{W}\{K(Z, W), \phi(W)\},
$$

where the subscript $W$ of $D$ denotes the variable with respect to which differentiation and integration are to be carried out. The characteristic property (8) of reproducing every function of class $\Gamma$ after scalar multiplication determines the kernel function in a unique way, as may easily be shown. Therefore, the kernel function $K(Z, W)$ is independent of the particular orthonormal set $\left\{\phi_{\nu}(Z)\right\}$ from which it was constructed. This fact proves of great importance in the theory of orthonormal functions and permits numerous applications.

The significance of the kernel function was discussed in our previous paper and we proved the identity

$$
K(Z, W)=\frac{1}{2 \pi}[N(Z, W)-G(Z, W)] .
$$

From (9) it follows that $K(Z, W)$ is a symmetric regular function of $Z$ and $W$ and a solution of (1) which has on $C$ the same boundary values as $(1 / 2 \pi) N(Z, W)$ and the same normal derivatives as $-(1 / 2 \pi) G(Z, W)$.

${ }^{3} \mathrm{~S}$. Bergman, On functions satisfying partial differential equations of elliptic type and their representation, Duke Math. J. vol. 14(1947) pp. 349-366. 
We proved further: If $f(Z)$ is a continuously differentiable function of $Z$ except for isolated points where it may have logarithmic infinities and if $D\{f, f\}<\infty$ the function

$$
\psi(Z)=D_{W}\{K(Z, W), f(W)\}
$$

is a regular solution of (1) which has on $C$ the same boundary values as $f(Z)$. This result leads at once to the construction of Green's function if an arbitrary fundamental solution is known. For if. $S(Z, W)$ is this fundamental solution,

$$
\sigma(Z, W)=D_{T}\{K(Z, T), S(T, W)\}
$$

is a solution of (1) of the class $\Omega$ having for $Z \in C$ the same values as $S(Z, W)$. Hence

$$
G(Z, W)=S(Z, W)-\sigma(Z, W)
$$

is just the required Green's function.

In this way we effect the construction of Green's function by means of an arbitrary fundamental solution and the kernel function. In view of (9) this leads to an explicit expression of Neumann's function, too. It is, however, often very difficult to obtain any fundamental solution $S(Z, W)$ and we shall therefore give in the following sections a construction of the Neumann function which does not presuppose the knowledge of any fundamental solution.

3. An identity for the Neumann function. Let $f(Z)$ be continuously differentiable in $B$, with the possible exception of a finite number of points where it may have logarithmic infinities. Let further $D\{f, f\}<\infty$. We suppose at first that $f(Z)$ has on the boundary $C$ of $B$ continuous boundary values. By virtue of Green's identity we have then

$$
\begin{aligned}
D_{W}\{N(Z, W), f(W)\}= & -\oint_{C} f(W) \frac{\partial}{\partial n_{W}} N(Z, W) d s_{W} \\
& -\sum_{\mu=1}^{r} \oint_{k_{\mu}(\rho)} f(W) \frac{\partial}{\partial n_{W}} N(Z, W) d s_{W}+\epsilon(\rho)
\end{aligned}
$$

where the $k_{\mu}(\rho)$ denote small circles of radius $\rho$ around the point $W=Z$ and the infinities of $f(Z)$, and where all integrations are taken in the positive sense with respect to the domain $B$ from which the circles $k_{\mu}(\rho)$ have been eliminated. The term $\epsilon(\rho)$ converges to zero if $\rho$ does so. Because of the symmetry of the Neumann function in $Z$ and $W$ and in view of the vanishing of its normal derivative on $C$, the first integral on the right-hand side is zero. If we let $\rho \rightarrow 0$, all the 
integrals around the infinities of $f(z)$ converge to zero, while the integral around the point $Z=W$ tends to the limit $-2 \pi f(Z)$. Hence we get in the limit $\rho=0$ finally

$$
D_{W}\left\{\frac{1}{2 \pi} N(Z, W), f(W)\right\}=f(Z)
$$

for every function $f(Z)$ satisfying the aforementioned quite general conditions.

We may even drop the assumption on the boundary values of $f(Z)$ on $C$. For though it played a certain part during the proof, the boundary $C$ has vanished in the final identity (13). We may approximate more general functions $f(Z)$ in $B$ by functions which have continuous boundary values and by the usual considerations extend the results to the class $\Lambda$ of all functions $f(Z)$ which have continuous derivatives in $B$ except for a finite number of logarithmic infinities and for which the Lebesgue integral $D\{f, f\}$ converges.

We see from (13) that the Neumann function $N(Z, W)$ has with respect to the class $\Lambda$ of continuously differentiable functions with a finite number of logarithmic infinities in $B$ just the same characteristic property as has the kernel function (cf. (8)) with respect to the class $\Gamma$ of solutions of (1). One might therefore expect that the Neumann function appears as a kernel function of a certain orthonormal set of functions of class $\Lambda$. A certain difficulty arises, however, from the possible logarithmic infinities of the functions considered and the logarithmic pole of $N(Z, W)$ itself. This fact leads to a certain complication in all our considerations.

4. The construction of the Neumann function by means of an orthonormal set. Consider the class $\Omega$ of all functions $g(Z)$ which have continuous derivatives in $B$ and for which $D\{g, g\}<\infty$. Obviously $\Omega \subset \Lambda$. Let $\left\{g_{\nu}(Z)\right\}$ be a complete orthonormal set of the class $\Omega$. In the case of a finite smoothly-bounded domain $B$, the existence of such a system is ensured by Weierstrass' approximation theorem, and it may even be chosen as a sequence of polynomials in $x$ and $y$.

Since the Neumann function becomes infinite for $Z=W$, we have no possibility of developing it into a series of the $g_{\nu}(Z)$ which converges in every closed subdomain of $B$. But if we denote Green's function of $B$ with respect to the ordinary Laplace equation by $\gamma(Z, W)$, we find immediately from (3) and $\left(3^{\prime}\right)$ that

$$
l(Z, W)=N(Z, W)-\gamma(Z, W)
$$


belongs to the class $\Omega$. In fact, $N(Z, W)$ being a fundamental solution of (1), we may write

$$
l(Z, W)=[A(Z, W)-1] \log \frac{1}{|z-w|}+\beta(Z, W)
$$

where $\beta(Z, W)$ is of class $\Omega$. The term $A(Z, W)-1$ vanishes for $Z=W$ at least to the second order; for, upon introducing $N(Z, W)$ into the equation (1) and using the fact that $A(Z, W)$ is itself a solution of (1), one finds

$$
\frac{\partial A(Z, W)}{\partial x} \frac{x-u}{|z-w|^{2}}+\frac{\partial A(Z, W)}{\partial y} \frac{y-v}{|z-w|^{2}}=\chi(Z, W)
$$

where $\chi(Z, W)$ remains finite as $Z \rightarrow W$. Hence, one concludes easily that

$$
\frac{\partial A(Z, W)}{\partial x}=O(|z-w|), \quad \frac{\partial A(Z, W)}{\partial y}=O(|z-w|) .
$$

From $\left(3^{\prime}\right)$ and $\left(14^{\prime \prime \prime}\right)$ we recognize at once that $l(Z, W)$ is of class $\Omega$.

Let us compute now the Fourier coefficients of $l(Z, W)$ with respect to the set $\left\{g_{\nu}(W)\right\}$. In view of (13) and (14) we obtain

$$
\begin{aligned}
c_{\nu}(Z) & =D_{W}\left\{l(Z, W), g_{\nu}(W)\right\} \\
& =2 \pi g_{\nu}(Z)-D_{W}\left\{\gamma(Z, W), g_{\nu}(W)\right\} .
\end{aligned}
$$

We associate with every function $g(Z)$ of class $\Omega$ a transformed function

$$
h(Z)=-\frac{1}{2 \pi} D_{W}\{\gamma(Z, W), g(W)\} .
$$

Then we may write instead of (15)

$$
c_{\nu}(Z)=D_{W}\left\{l(Z, W), g_{\nu}(W)\right\}=2 \pi\left[g_{\nu}(Z)+h_{\nu}(Z)\right]
$$

where the $h_{\nu}(Z)$ are associated with $g_{\nu}(Z)$ by (16).

One proves in the usual way the Parseval equality

$$
\sum_{\nu=1}^{\infty} c_{\nu}(Z)^{2}=4 \pi^{2} \sum_{\nu=1}^{\infty}\left[g_{\nu}(Z)+h_{\nu}(Z)\right]^{2}=D_{W}\{l(Z, W), l(Z, W)\}
$$

and obtains in particular the convergence of the left-hand sum.

Consider next the function $l(Z, W)+l(T, W)$ which is, as a function of $W$, of class $\Omega$. Its Fourier coefficient with respect to $g_{\nu}(W)$ is obviously $c_{\nu}(Z)+c_{\nu}(T)$. Applying now the Parseval equality with 
respect to this function, we find

(18) $\sum_{\nu=1}^{\infty}\left[c_{\nu}(Z)+c_{\nu}(T)\right]^{2}=D_{W}\{l(Z, W)+l(T, W), l(Z, W)+l(T, W)\}$.

By a simple transformation and using the identity (17), we obtain

$$
\sum_{\nu=1}^{\infty} c_{\nu}(Z) c_{\nu}(T)=D_{W}\{l(Z, W), l(T, W)\} .
$$

The left-hand sum converges in view of the convergence of the sum (17) and of the Schwarz inequality.

This result may be simplified again by using the definition (14) of $l(Z, W)$ and the identity (13). We find easily

$$
\begin{aligned}
\sum_{\nu=1}^{\infty} c_{\nu}(Z) c_{\nu}(T)= & 2 \pi N(Z, T)-4 \pi \gamma(Z, T) \\
& +D_{W}\{\gamma(Z, W), \gamma(T, W)\} .
\end{aligned}
$$

We transform further the last term on the right-hand side. By definition (2) and Green's identity one obtains almost immediately (with $\Delta_{W}=\partial^{2} / \partial u^{2}+\partial^{2} / \partial v^{2}$ )

$$
\begin{gathered}
D_{W}\{\gamma(Z, W), \gamma(T, W)\}=2 \pi \gamma(Z, T)-\oint_{C} \gamma(Z, W) \frac{\partial \gamma(T, W)}{\partial n_{W}} d s_{W} \\
-\iint_{B} \gamma(Z, W)\left[\Delta_{W} \gamma(T, W)-P_{\gamma}(T, W)\right] d u d v
\end{gathered}
$$

whence, by the definition of Green's function $\gamma(Z, W)$ of the Laplace equation,

$$
\begin{aligned}
D_{W}\{\gamma(Z, W), & \gamma(T, W)\} \\
& =2 \pi \gamma(Z, T)+\iint_{B} P(W) \gamma(Z, W) \gamma(T, W) d u d v .
\end{aligned}
$$

From $\left(15^{\prime}\right),(20)$ and $\left(21^{\prime}\right)$ we obtain finally the following representation for the Neumann function

$$
\begin{aligned}
N(Z, T)= & \gamma(Z, T)-\frac{1}{2 \pi} \iint_{B} P(W) \gamma(Z, W) \gamma(T, W) d u d v \\
& +2 \pi \sum_{\nu=1}^{\infty}\left[g_{\nu}(Z)+h_{\nu}(Z)\right]\left[g_{\nu}(T)+h_{\nu}(T)\right] .
\end{aligned}
$$

On the right-hand side of this equation occur terms which are not 
directly connected with the differential equation (1). One has only Green's function for the Laplace equation, a complete orthonormal set for the class $\Omega$, and certain other expressions which are obtained from the foregoing by simple processes of differentiation and integration. (22) represents the main result of this paper. It permits an effective calculation of Neumann's function with respect to the partial differential equation (1) by means of a complete set of functions of class $\Omega$ orthonormalized by the Dirichlet integral (2) connected with that differential equation.

5. The construction of the Green function by means of an orthonormal set. Since Green's function is related by (9) with the kernel function and the Neumann function, we solved in the preceding paragraph also the problem of constructing Green's function by orthonormal functions. We may, however, find a procedure which permits us to find Green's function directly in terms of a particular orthonormal set.

For this purpose consider the expression

$$
D_{W}\{G(Z, W), f(W)\}=F(Z)
$$

where $f(W)$ is an arbitrary function of the class $\Lambda$ defined in $\S 3$. This expression may be transformed by means of (9), (10) and (13); one obtains easily

$$
F(Z)=2 \pi[f(Z)-\psi(Z)]
$$

where $\psi(Z)$ is that function of class $\Gamma$ which has on $C$ the same boundary values as $f(Z)$.

We define the class $\Omega^{\circ}$ of functions $f^{\circ}(Z) \in \Omega$ which have on $C$ the boundary value 0 . From (23) and (24) we conclude

$$
D_{W}\left\{\frac{1}{2 \pi} G(Z, W), f^{o}(W)\right\}=f^{o}(Z), \quad \text { for } f^{o}(Z) \in \Omega^{o},
$$

since the only function of class $\Gamma$ which vanishes on $C$ is the constant zero.

Green's function has with respect to the class $\Omega^{\circ}$ the characteristic property of reproducing each function of this class by scalar multiplication. We may, therefore, construct Green's function by means of a complete orthonormal system with respect to this class in exactly the same way as we did for Neumann's function by means of a complete orthonormal system with respect to $\Omega$. In fact, let $\left\{g_{\nu}^{o}(Z)\right\}$ be a complete orthonormal system with respect to $\Omega^{\circ}$ and $\left\{h_{\nu}^{o}(Z)\right\}$ the set of functions associated with it by means of (16). 
Then, the conclusions of $\$ 4$ lead now to the formula

$$
\begin{aligned}
G(Z, T)= & \gamma(Z, T)-\frac{1}{2 \pi} \iint_{B} P(W) \gamma(Z, W) \gamma(T, W) d u d v \\
& +2 \pi \sum_{\nu=1}^{\infty}\left[g_{\nu}^{o}(Z)+h_{\nu}^{o}(Z)\right]\left[g_{\nu}^{o}(T)+h_{\nu}^{o}(T)\right]
\end{aligned}
$$

since $l^{\circ}(Z, W)=G(Z, W)-\gamma(Z, W)$ obviously belongs to the class $\Omega^{o}$.

Thus we may summarize our results in the following way:

I. Consider the sub-class $\Gamma$ of $\Omega$ consisting of the solutions of (1). The kernel function of any complete orthonormal system with respect to $\Gamma$ is $K(Z, W)$.

II. Consider the class $\Omega^{\circ}$ of all functions $f^{\circ} \in \Omega$ which vanish on the boundary $C$. Constructing the kernel function of any complete orthonormal system with respect to $\Omega^{\circ}$ and taking the necessary steps in order to guarantee convergence leads to Green's function.

III. Carrying out the same processes as in II for a complete orthonormal system with respect to the general class $\Omega$ leads to Neumann's function.

The relation (9) between the kernel function and the Green and Neumann functions appears now as a simple consequence of the fact that the linear space $\Omega$ is the sum of the two complementary orthogonal spaces $\Omega^{\circ}$ and $\Gamma$. Thus, we may construct a complete orthonormal system in $\Omega$ by combining any complete orthonormal system in $\Omega^{\circ}$ with another in $\Gamma$. The kernel function in $\Omega$ appears, therefore, as the sum of the two kernel functions in $\Omega^{\circ}$ and $\Gamma$.

A particular method for obtaining a complete orthonormal system for the class $\Omega^{\circ}$ is given by the following procedure. Consider the differential equation

$$
\frac{\partial^{2} u}{\partial x^{2}}+\frac{\partial^{2} u}{\partial y^{2}}-P u+\lambda u=0 .
$$

Determine the eigen values $\lambda_{\nu}$ for which $\left(1^{\prime}\right)$ has eigen functions $u_{\nu}(Z)$ which vanish on the boundary $C$ of $B$. We suppose for sake of simplicity that all eigen values $\lambda_{\nu}$ are nondegenerate. By Green's identity one has for each pair of eigen functions

$$
D\left\{u_{\nu}, u_{\mu}\right\}=-\iint_{B} u_{\nu}\left[\Delta u_{\mu}-P u_{\mu}\right] d x d y=\lambda_{\mu} \iint_{B} u_{\nu} u_{\mu} d x d y .
$$

In view of the symmetry of $D\left\{u_{\nu}, u_{\mu}\right\}$ and of the fact that $\lambda_{\nu} \neq \lambda_{\mu}$ for 
$\nu \neq \mu,(26)$ implies the orthogonality of the eigen functions in our metric. Assuming the eigen functions normalized in the usual way,

$$
\iint_{B} u_{\nu}^{2} d x d y=1
$$

we have in

$$
v_{\nu}(Z)=\frac{1}{\lambda_{\nu}^{1 / 2}} u_{\nu}(Z)
$$

an orthonormal system in our metric.

By a classical result of the theory of integral equations, all $v_{\nu}(Z)$ form a complete system in $\Omega^{\circ}$. Hence this orthonormal system may be used for the construction of the the Green function. This particular result leads to a well known theorem in the theory of eigen functions.

6. Discussion of the result. In order to understand the significance of our result, we have to investigate the meaning of the terms $g_{\nu}(Z)+h_{\nu}(Z)$. By definition $(16), h_{\nu}(Z)$ is of class $\Omega$ and has the boundary values 0 . One easily shows further:

$$
\Delta h_{\nu}(Z)=P(Z) g_{\nu}(Z)-\Delta g_{\nu}(Z), \quad \Delta=\frac{\partial^{2}}{\partial x^{2}}+\frac{\partial^{2}}{\partial y^{2}} .
$$

Hence $g_{\nu}(Z)+h_{\nu}(Z)$ is a function of class $\Omega$ with the same boundary values on $C$ as $g_{\nu}(Z)$ and satisfying in $B$ the differential equation

$$
\Delta\left[g_{\nu}(Z)+h_{\nu}(Z)\right]=P(Z) g_{\nu}(Z) .
$$

This fact clarifies the meaning of (22). For $N(Z, T)$ satisfies as function of each argument the differential equation (1). If we apply in (22) the Laplace operator with respect to $Z$ and $T$ on both sides, we obtain formally

$$
P(Z) P(T) N(Z, T)=P(Z) P(T) \cdot 2 \pi \sum_{\nu=1}^{\infty} g_{\nu}(Z) g_{\nu}(T) .
$$

If the right-hand side sum were convergent, this would give a representation of the Neumann function as the kernel function with respect to the class $\Omega$. In order to ensure convergence, however, we had to introduce the $g_{\nu}(Z)+h_{\nu}(Z)$, that is, to carry out a certain integration of the formal identity $\left(22^{\prime}\right)$. Analogous considerations are valid in the case of formula (25) and the theory of Green's function.

One might inquire further concerning the importance of the Green's function $\gamma(Z, W)$ in our considerations. We used essen- 
tially the two following properties of $\gamma(Z, W)$ :

(a) $\gamma(Z, W)$ has at $Z=W$ a logarithmic pole;

(b) $\gamma(Z, W)$ is a symmetric harmonic function;

while the third and most restrictive property of $\gamma(Z, W)$, that is, that it vanishes on the boundary $C$ of $B$, was only used in the transformation of (21) into $\left(21^{\prime}\right)$. It might be more convenient for practical computations to use instead of $\gamma(Z, W)$ simply the singularity function $-\log |z-w|$ which satisfies both conditions (a) and (b). The representation of the Neumann function is in this case a little more complicated, but on the other hand one is saved the effort of computing the Green's function of the domain with respect to Laplace's equation.

Finally, we wish to point out that we dealt with a particular type of the partial differential equation (1) only for the sake of a simple representation of the method. We indicated in our previous paper the possible generalizations of the type of equation, and for all those partial differential equations obtained one may construct the Green and Neumann functions in an analogous way.

HARVARD UNIVERSITY

\section{THE ISOPERIMETRIC PROBLEM OF BOLZA WITH FINITE SIDE CONDITIONS}

J. ERNEST WILKINS, JR.

1. Introduction. In view of recent improvements in the theory of the problem of Bolza in the calculus of variations, both with respect to the necessary $[2,6]^{1}$ and the sufficient conditions $[3,4,5,7]$, it seems worthwhile to investigate certain problems which, although not immediate special cases of the problem of Bolza, can be transformed by simple means into such problems. A comparatively simple problem of this nature is that of finding necessary conditions and sufficient conditions in order that an arc

C:

$$
a_{h}, y_{i}(x)
$$$$
\left(h=1, \cdots, r ; i=1, \cdots, n ; x_{1} \leqq x \leqq x_{2}\right)
$$

will minimize an expression of the form

$$
I(C)=g(a)+\int_{x_{1}}^{x_{2}} f\left(a, x, y, y^{\prime}\right) d x
$$

Received by the editors April 3, 1947.

1 Numbers in brackets refer to the bibliography at the end of the paper. 